\title{
Coaching educativo: desarrollo de competencias en el educando de nivel superior
}

\section{Educational coaching: development of competences in higher education}

\author{
Luis Antonio Álvarez Silva
}

Hugo Santiago Izquierdo Lara

Universidad de Especialidades Espíritu Santo, Ecuador

Lurdes Sabrina Gudiño Gómez

María Mercedes Macías Montoya

Instituto Tecnológico Superior Vicente Rocafuerte, Ecuador

Autor para correspondencia: lalvarezsilva@uees.edu.ec; lgudino@itsvr.edu.ec; mmacias@itsvr.edu.ec; hizquierdo@uees.edu.ec

Fecha de recepción: 23 de agosto de 2018 Fecha de aceptación: 15 noviembre del 2018

Resumen: Los procesos de educación superior tienden a adoptar nuevas metodologías con el fin de potencializar las actividades de enseñanza - aprendizaje. El presente documento tiene como objetivo principal identificar el desarrollo de las competencias académicas a través de la implementación del proceso de Coaching educativo en el nivel superior. El diseño de la investigación obedece a una metodología experimental de tipo pre-experimental (estudio de caso). La muestra estuvo conformada por un total de 121 alumnos de la carrera contable de un Tecnológico público de la ciudad de Guayaquil-Ecuador. El Coaching, en general, es un proceso orientado en el desarrollo potencial del Coachee; además este proceso también puede abordarse desde otros escenarios, por ejemplo: el deporte, el trabajo, la familia, etc. Dentro de las conclusiones del estudio, se contempla que el rol del Coaching educativo tiene características transformadoras en el estudiante, ya que se orienta a la mejora de sus competencias.

Palabras clave: coaching; coaching educativo; educación; pedagogía; educación superior; competencias

\begin{abstract}
The processes of higher education tend to adopt new methodologies in order to potentiate the teaching - learning activities. The main objective of this document is to identify the development of academic competences through the implementation of the educational Coaching process at the higher level. The design of the research is based on an experimental methodology of a pre-experimental type (case study). The sample consisted of a total of 121 students in the accounting career of a public technologist in the city of Guayaquil-Ecuador. Coaching, in general, is a process oriented towards the potential development of the Coachee; In addition, this process can also be approached from other scenarios, for example: sports, work, family, etc. Within the conclusions of the study, it is contemplated that the role of educational Coaching has transforming characteristics in the student, since it is oriented towards the improvement of their competences.
\end{abstract} Key words: coaching; educational coaching; education; pedagogy; higher education; skills 


\section{Introducción}

El conocimiento es el resultado de una serie de situaciones positivas y negativas que le acontecen al sujeto, por consiguiente influyen en la sociedad en que este se encuentre. Es por ello que una de las etapas de mayor influencia en los sujetos es su educación, ya que esta se encuentra en continuo cambio y mejoramiento (González y Esteban, 2013). Es por ello, que la educación y la Pedagogía conlleva un rol predominante en la formación de los individuos.

La educación puede ser conceptualizada desde varios enfoques (Gómez, 2012), sin embargo, la educación siempre se encuentra en proceso de cambio que no siempre garantizan una mejora continua o significativa, por tal razón en más de una vez se ha vuelto objeto de observaciones; es así que, lo mismo acontece con los sujetos o actores sociales que son parte de ella, tal es el caso del cuerpo docente y el educando. Por consiguiente, son muchas las propuestas de mejora que se han planteado en el ámbito educativo, pero a su vez, muchas de ellas solo han sido de carácter simple, sin representar resultados fructíferos en nuestra sociedad; es por ello que en más de un intento estas propuestas no han logrado una consecución de éxito.

El proceso Coaching, no es relativamente nuevo; de hecho se viene abordando desde una perspectiva analógica, desde épocas lejanas; tal es el caso de la mayéutica de Sócrates; posterior a ello, por los años 30, se lo relacionaba como un medio de trasporte denominado Kocs; por consiguiente, el término tuvo su respectiva trascendencia lingüística por los años 60 al ser más utilizado en el aspecto deportivo; en sí, a finales de los años 80 se lo relaciona con el mundo empresarial y hoy por hoy incide en los sistemas educativos con un rol trasformador (Ruiz Fernández et al., 2014).

Este tema, que ha presentado avances significativos a nivel de investigaciones científicas y bibliográficas, ha repercutido en la elaboración de una serie de libros, artículos académicos, programas de formación, presentación de varios autores con diferentes enfoques etc. Sin embargo es importante conocer las bases científicas del término, para su mejor compresión y análisis.

Para abordar la temática del Coaching educativo, es necesario el análisis de ciertos elementos que construyen el término, tal es el caso de la educación y la Pedagogía, con sus respectivos enfoques. Siguiendo este orden de ideas, El objetivo principal del presente artículo oscila en identificar el desarrollo de competencias académicas a través de la implementación del Coaching educativo en los procesos de enseñanza aprendizaje del educando de nivel superior. Adicionalmente, se analizarán los factores teóricos y culturales de la educación, pedagogía y Coaching educativo en el entorno educativo.

\section{Fundamentación teórica}

\section{Educación}

El rol de la educación en la sociedad tiene un carácter primordial, claro está que esto depende mucho la postura social, política y económica de los pueblos (Recalde et al., 2013). Sin embargo, es importante recalcar que la educación puede ser enfocada desde tres ángulos, es decir puede ser vista como: un sistema social, ejemplo de ello sería la educación norteamericana, la 
educación occidental; que la encasilla como el resultado de una acción, ejemplo de ello sería un modelo de educación progresista, tecnológica; y por último como un proceso entre dos o más sujetos encaminados a un fin (Gómez, 2012).

Por esta razón, se puede definir a la educación como un proceso de interacción entre sujetos que tienen como efecto la adquisición de conocimientos, valores, experiencias, competencias y desarrollo personal (Vásquez, 2012). Por ende, la relación que conllevan dichos sujetos debe poseer particularidades que permitan el logro de metas y objetivos. Por esto, se puede considerar que la mayor cantidad de objetivos trazados, se logran cuando el sujeto tiene un mayor grado de estabilidad emocional y cognitiva. Por consiguiente, esto sería en la etapa de juventud y adultez, que es cuando cursa un nivel superior de estudios y su motivación lo impulsa de una forma determinada (Ruiz et al., 2012).

La práctica educativa orientada a la reflexión y a la mejora de los procesos de enseñanza aprendizaje, es de suma importancia hoy en día, es por ello que los docentes, alumnos y directivos deben orientarse a la innovación y cambios de los procesos con una proyección de mejora continua (Arzate, 2013). Para esto, es necesario analizar los avances a nivel de enseñanzas, aprendizajes, metodologías innovadoras, evaluaciones cuantitativas, cualitativas y acompañamiento estudiantil que están presentando los demás modelos educativos en otros países, tal es el caso de Finlandia.

Para el presente artículo, el enfoque primordial de la educación es el procesal. Por consiguiente el sumario que se conlleve con los actores sociales de la educación es fundamental para su desarrollo. El ámbito de la educación en muchos países circula en tres o cuatros categorías, estas son: la educación primaria, la educación secundaria, la educación superior (profesionalización) y la educación de cuarto nivel (postgrado o maestría). Siguiendo este orden de ideas, es importante señalar el papel que la educación ejerce en el sujeto que está en una etapa superior; ya que en esta etapa, por lo general, el sujeto tiene definida sus metas profesionales para aportar a la sociedad desde una perspectiva de mayor conocimiento y que se evidencia en su evaluación (Cubero et al., 2017).

\section{Pedagogía}

Desde un punto de análisis tradicional y contemporáneo, la Pedagogía está en unidad indisoluble a la educación. Sin embargo, esta ha presentado varios enfoques para abordar ciertas temáticas de gran impacto, tal es el caso que la Pedagogía se la puede definir desde un enfoque de: reflexión, práctica, teoría y ciencia (Bravo, 2013).

Por consiguiente, si se define a la Pedagogía como aquella ciencia que tiene como objeto de estudio la educación, se estaría dando una perspectiva reduccionista, ya que eso conllevaría a que la Pedagogía mantenga una dependencia a otras ciencias tales como la Psicología, Sociología, Antropología, entre otras. Siguiendo este orden de ideas y para el desarrollo del presente artículo; la Pedagogía, como ciencia con base filosófica, es considerada como aquel saber que estudia la "formación" del sujeto para que este sea un constructor y transformador de la realidad (Ospina, 2013). 
Por tal razón, el enfoque formador de la Pedagogía es muy relevante para la temática del Coaching Educativo. La práctica Pedagógica es la consecución de aquel objeto de estudio que se mencionó en el párrafo anterior, por ello es fundamental que se defina como aquella que conlleva a la formación del sujeto. Además, la Pedagogía posee modelos, prácticas, enfoques, entre otros, que han permitido su crecimiento, desarrollo y aportes a otras disciplinas y ciencias del saber.

Los modelos Pedagógicos tradicionales más importantes son tres; el modelo conductista el cual señala que la formación del sujeto está alineada a un cambio conductual (estimulorespuesta); el modelo socio-histórico-cultural, que señala que la formación y aprendizaje de del individuo está alineado a un condicionamiento social, es decir influenciado por el medio/cultura, y que el proceso de interiorización esta defino por la ZDP (zona de desarrollo próximo). Por último, el modelo constructivista, el cual está enfocado en un aprendizaje procesal, es decir, el aprendizaje de un determinado sujeto se desarrolla por medio de procesos de transformación mentales constructivas y ordenadas (Ortiz et al., 2015).

De forma contemporánea ha surgido un nuevo modelo pedagógico, que es la Pedagogía dialogante, la cual señala que el objeto de la pedagogía no es la enseñanza (como señalan algunas escuelas tradicionales) sino el "desarrollo" cognitivo, valorativo y práctico de los sujetos, a través de los medios que la formación proporcione (Valer \& Hernández, 2010).

En fin, ninguno de los cuatro modelos mencionados, se lo puede considerar como único o más efectivo; ya que sobre toda circunstancia de educación están inmersos factores tales como la metodología de aprendizaje, los objetivos a alcanzar, la realidad social, la política educativa, cambios radicales a nivel país, etc.

Siguiendo este orden de ideas; la Pedagogía como tal, es una ciencia que influye de manera directa en los procesos de enseñanza, desarrollo, aprendizaje y formación, por esta razón influye en los procesos de Coaching, ya que la relación entre los miembros del proceso Coaching tiene como fin un desarrollo formativo, a diferencia que éste no está centrado en las soluciones que dé quien gestiona el proceso, más bien está centrado en la formación que tenga el sujeto que auto gestiona el proceso; esto se analiza en los siguientes párrafos.

\section{Coaching}

En la actualidad es muy común leer y escuchar de la terminología Coaching; esto se debe al gran impacto que este fenómeno ha tenido en los últimos años (Sandoval y López, 2017), no sólo en los ámbitos deportivos y empresariales sino también en otras áreas de la vida cotidiana, tal es el caso de la educación.

Es necesario utilizar referencias históricas para aterrizar el tema a nuestra actualidad y a una adecuada definición. Por ello, es importante considerar que, desde un punto de vista filosófico, uno de los orígenes del Coaching fue en la Mayéutica de Sócrates; ya que este filosofo utilizaba un modelo adecuado de hacer preguntas, para que así sus discípulos puedan "sacar a relucir" lo que llevan consigo mismo. Desde un enfoque Psicológico, tenemos el Humanismo de Carl Rogers, quien tomaba de referencia conceptos de voluntad, autorrealización y el nivel de alta potencialidad del sujeto (Sans, 2012). 
Es considerable mencionar, que el Coaching no es unidireccional ni solo tiene una metodología de aplicación, tampoco tiene un creador único, tampoco ninguna escuela en el mundo puede denominarse la verdadera. De hecho, el Coaching es un nombre muy utilizado y que según la escuela y el autor que lo teoriza, se define de diferente forma, sin embargo se puede señalar que ha recibido grandes aportes de la Psicoterapia, Psicología y el asesoramiento (Gorrochotegui-Martell, 2011).

El origen del vocablo Coaching posee dos versiones, que en efecto son europeas. La primera es de origen Húngaro, por la utilización de un carruaje en la ciudad de Kocs al cual se denominaba "carruaje de Kocs" el cual servía como medio para la transportación de personas a diferentes lugares. Posterior a ello, el vocablo paso a otros países y tomo diferencia en la forma de escritura y pronunciación ejemplo de ello fue lo que paso en Alemania en el que se denominó kutsche, en Italia donde se denominó cocchio y en España donde se estableció como coche. La segunda versión del término Coaching oscila en Inglaterra, que resulta de sustantivar el verbo to Coach, que su traducción al idioma español significaría entrenar (Veliz y Paravic, 2012)

Continuando este orden de ideas, se puede decir que no hay una definición específica para la palabra Coaching, esta depende de quien lo hace, cómo lo hace y en que fundamenta su gestión. Para adentrar más en esta temática hay que considerar que en la actualidad existen grandes entidades mundiales de Coaching; tal es el caso de la Internacional Coach Federation (ICF) que define el Coaching como una relación profesional continua y temporal que tiene un mutuo acuerdo entre los integrantes; la Escuela Europea de Coaching lo define como el arte de plantear preguntas para poder ayudar a las personas por medio de la revelación de nuevas formas de abordaje y que conllevan a alcanzar objetivos (Sans, 2012). Otras definiciones señalan al Coaching como una herramienta, técnica, conversatorio, dialogo, etc.

Dos de los autores más reconocidos en la temática, Gallwey y Whitmore señalan que el Coaching consiste en la liberación del potencial de un individuo con el fin de aumentar a grandes niveles su desempeño, en la que el aprendizaje sustituye a la enseñanza. (Whitmore, 2003). En sí, según las revisiones realizadas en autores tales como Bayón, 2006; Bisquerra, 2008; Cantera, 2003; Gordillo, 2008; Launer, 2007; se sintetiza que el Coaching es el proceso de interacción sistemática ente dos o más individuos, en el cual está inmerso el aprendizaje mutuo y el desarrollo del potencial individual que tiene como frontera la mejora continua, citado por (Sánchez y Boronat, 2014).

En el proceso de Coaching la comunicación juega un papel trascendental, ya que es uno de los medios más fuertes que sistematiza la actividad (Giménez et al.2016), por ello los roles que existen en este proceso son el "Coach" que se lo podría definir como el entrenador, modelador y/o guía; y el "Coachee" que hace referencia al cliente, usuario y/o alumno (Piñeiro et al.2013).

\section{Coaching educativo}

Según Bayo, 2006; el Coach puede desarrollar roles en relación al Coachee; por ello, este puede ejercer la función de maestro por su capacidad de análisis; de socio por su habilidad de proyectar estrategias; de investigador por su ímpetu en descubrir situaciones; de profesor porque incita al criterio; y como monitor de avances planeados, citado por (Sánchez y Boronat, 2014) 
Normalmente se tiene habituado que los procesos de Coaching se dan en el campo empresarial (Musicco, 2015) y/o en el ámbito de la formación deportiva (Sánchez et al., 2017), por la basta información de ello y por los resultados a gran escala, ya que ello ha favorecido el desarrollo personal y/o profesional de un sujeto que proyecta y cumple sus metas (SánchezTeruel, 2013). Sin embargo, las metas de un sujeto no solo son a nivel empresarial y/o deportivo, ya que como se menciona en el párrafo anterior en el campo educativo también puede existir Coaching (Gorrochotegui-Martell et al., 2014).

Como señala Bou (Bou-Pérez, 2007), El Coaching a nivel educativo se lo puede definir como el proceso (conjunto de etapas) sistémico orientado al aprendizaje y al accionar, que tiene bases en el presente pero se proyecta al cambio, y es en este proceso que se aplican herramientas y se utilizan recursos que ayudan a mejorar el resultado y el desempeño de los individuos que lo aplican, citado por (Lárez, 2008). Martínez, aporta otra definición al Coaching educativo, aquel lo define como el proceso formal de instruirse, encaminado a que el sujeto aprenda por sí mismo a descubrir y equilibrar situaciones relacionadas a su forma de pensar, sentir, comportarse e interactuar, con el fin de obtener un auto cambio de carácter transformador. (Veliz y Paravic, 2012).

La definición del Coaching educativo está afinada a ser un proceso de cambio transformador, en el cual el rol del aprendizaje y de la pedagogía juegan un papel crucial, ya que ambas son adherentes a la formación del individuo; por tal razón entiéndase individuo a todos los actores sociales que intervienen en el proceso, es decir; el alumno, el profesor y todos aquellos inmerso en el proceso (López Villafranca y Gómez de Travesedo, 2016).

El presente artículo, se enfoca en el desarrollo del estudiante como tal, por tal razón la dinámica del tema Coaching, está orientado al Coaching como servicio pedagógico encaminado a mejorar las competencias del educando de nivel superior; por esta razón el estudiante posee un rol de Coachee y el docente de Coach.

En sí, Bou (2007) señala que la fundamentación del adecuado Coaching educativo se basa en tres aspectos esenciales; estos son: los objetivos, las creencias y los valores. Los objetivos hacen referencia a la situación o meta deseada; las creencias hacen mención a todos aquellos constructos mentales que sirven de guía a nuestro modo de vida, que de alguna u otra manera delimitan la forma de pensar, actuar y sentir; por tal razón una de las luchas en el Coaching es que aquella forma de pensar, actuar y sentir no influya negativamente en el proceso, sino que se convierta en factores positivos; por último están los valores, estos son los elementos que definen a cada sujeto y que están de forma inherente en el proceso para obtener un efecto de satisfacción (Pimentel y Rodríguez, 2016).

Entre los aspectos más elementales que se pueden presentar en el proceso de Coaching educativo, están las reuniones y conversaciones que tenga el Coach (docente) y el Coachee (alumno); para lo cual deben estar definidos los siguientes aspectos: la diferencia de roles, adecuado ambiente para dialogar, la corresponsabilidad en el aprendizaje, los objetivos estratégicos del Coachee, la meta, la forma de realizar el accionar, disposición de 
implementación de nuevas estrategias, libertad para la toma de decisiones que proporcionen beneficio, apoyo del Coach, interacción de carácter dinámico, etc. (Malagón, 2011).

Las cualidades que debe tener un docente Coaching, es con base a sus competencias técnicas (conocimiento) y conductuales (comportamientos), por tal razón Mañez, Navarro y Bou en 2007 en su escrito Coaching para docentes hacen hincapié en que las competencias técnicas que debe tener un Coach educativo son: visión, sabiduría, comunicación eficaz; y dentro de las competencias conductuales, están: humildad, flexibilidad, seguridad propia, paciencia, comunicación asertiva, consistencia, coherencia, convicción, proactividad e inteligencia emocional (Máñez et al., 2008).

Estas competencias, son adquisiciones y formaciones; producto del conocimiento, desarrollo de habilidades y práctica que tenga el Coach que guía a la acción y la enseñanza de los sujetos (Isidoria et al., 2015). Estas, se pueden obtener por medio de capacitación especializada en entidades avaladas y que permitan obtener licencias certificadas en Coaching, tales como: Escuela de Coaching Europeo (EUC), la International Coach Federation (ICF), la Asociación Española de Coaching (ASESCO), entre otras (Sans, 2012).

\section{Métodos}

La presente información es producto de un proceso de investigación experimental de tipo pre experimental o también denominada estudio de caso, en el cual la variable dependiente es el desarrollo de las competencias del educando (Hernández Sampieri et al., 2014). Para su realización, se partio de los resultados obtenidos de la evaluación de $360^{\circ}$ realizada a los alumnos que cursaron el 2 do semestre de la carrera Administrativa Contable; esto fue, en el cierre del 1er periodo académico 2017. Posterior a ello, se contrastó con los resultados de la evaluación de $360^{\circ}$ del siguiente periodo. Todo con el debido consentimiento escrito de las autoridades y alumnado.

Las competencias, objeto de medición de la institución son: Adaptabilidad, que es aquella competencia o capacidad que permite al educando identificar y comprender los sucesos y cambios del entorno para transformar debilidades en fortalezas a través de la acción; Trabajo en equipo, como aquella capacidad para interactuar y colaborar con otros individuos con el objetivo de lograr una meta en común; Disposición al aprendizaje, que es aquella competencia orientada a la valoración del aprendizaje como elemento de desarrollo académico y profesional adoptando opciones de mejora; Orientación a los resultados, que es aquella capacidad de auto guiar conductas y/o de guiar la de otros sujetos al progreso de los resultados preestablecidos; y por último Comunicación, que comprende la capacidad de escuchar, entender y transmitir de forma eficaz y oportuna información a los intervinientes en el proceso de enseñanza - aprendizaje (Alles, 2009).

\section{Muestra}

La muestra está conformada por 121 alumnos que conforman el 3er semestre de la carrera Administrativa Contable, la misma que está dividida en 3 paralelos de 40, 38 y 43 alumnos, respectivamente. La elección de la muestra obedece a un muestreo por conveniencia, en la que el criterio de inclusión es la presencia regular en uno de los paralelos en mención (Hernández Sampieri et al.2014). Según el muestreo, El 67\% son mujeres mientras el 33\% son hombres; el 
$64 \%$ labora y el 36\% no labora; el 52\% son responsables de hogar y el $48 \%$ no; el promedio de edad de la muestra es de 24 años. Adicionalmente, para el presente estudio se consideraron los paralelos nocturnos (2) y vespertino (1).

\section{Procedimiento}

Una vez cerrado el primer periodo académico 2017, se procedió a la realización de la evaluación $360^{\circ}$ al educando, la misma que conlleva el análisis de 5 competencias las cuales son: adaptabilidad, trabajo en equipo, disposición al aprendizaje, proactividad y comunicación. Posterior a ello, en el inicio del segundo proceso académico se realizó el proceso de Coaching educativo por medio de la intervención de 3 docentes certificados en Coach. En este proceso evidenció la apertura de sesiones con grupos de 10 estudiantes de forma semanal, en un periodo de 45 minutos aproximadamente y que las sesiones no intervenga en sus horas académicas (Rosa et al.2015). El proceso de Coaching educativo consistió en 5 etapas, en la que cada una de ellas, tiene sus respectivas actividades.

La etapa 1 (explorar); en esta etapa se puede identificar como se encuentra el estudiante ante su situación académica, enfocándose en conocer en qué área de su vida le gustaría profundizar, ejemplo de ello sería el desarrollo personal, la carrera, el ocio, etc.; para esta etapa una de las herramientas más utilizadas es la rueda de la vida, la misma que permite enfocarse en el área que más aprecie el estudiante. La etapa 2 (enfocar); en este nivel la fijación de metas académicas es evidente, para ello se proporcionó la herramienta de diseño de objetivos Smart (específico, medible, alcanzable, realizable y temporal), en la que el educando diseña por medio de esta herramienta, qué nivel de desarrollo académico y de competencias desea alcanzar desde una forma realista y con un límite de tiempo efectivo (Universidad de Castilla-La Mancha, 2016).

La etapa 3 (analizar) es una etapa situacional, en esta el estudiante analiza que sucesos positivos y/o negativos están inmersos en su camino del desarrollo académico, por esta razón la utilización de la herramienta DAFO o FODA (según el objetivo) brinda las opciones para que el educando puede definir los aspectos que lo limitan y/o lo potencializan; otra de las herramientas utilizadas fue la Ventana de Johari. La etapa 4 (planificar) consiste en el diseño de tareas de forma ordenada, con ello se pretende lograr los objetivos antes señalados; para ello la herramienta utilizada en esta etapa fue el esquema de Ishikawa, que permite al estudiante ordenar sus acciones en relación al tiempo y a los recursos utilizables (Universidad de Castilla-La Mancha, 2016).

La etapa 5 (actuar) es considerada la última etapa del proceso; sin embargo esto no representa el cierre, más bien es el momento de traducir todas las demás etapas en acciones concretas (García-Naveira, 2013). Para ello, son relevantes las repeticiones de aquellas acciones, la visualización de éxito del estudiante y la automotivación del mismo (Universidad de CastillaLa Mancha, 2016).

El proceso de Coaching educativo, oscilo en un periodo de intervención de 4 meses por cada uno de los tres paralelos anteriormente mencionados; estos pertenecen a la carrera Administrativa Contable del Tecnológico Vicente Rocafuerte. En vísperas del cierre del 2 do proceso académico 2017, se procedió a la realización del proceso de evaluación $360^{\circ}$, como 
normativa de la misión y la calidad de la institución (Instituto Tecnológico Superior Vicente Rocafuerte, 2017).

\section{Resultados}

En el siguiente punto, se detallan los resultados obtenidos en el 1er periodo académico 2017 por medio de la evaluación $360^{\circ}$; versus los resultados del 2 do periodo académico 2017 con la utilización de la misma herramienta propia de la institución (Ver tabla 1 y 2).

Tabla 1.

\begin{tabular}{lccccc}
\hline n=121 & $\begin{array}{c}\text { Evaluación } \\
\text { docente }\end{array}$ & Autoevaluación & Heteroevaluación & Coevaluación & Promedio \\
\hline $\begin{array}{c}\text { Adaptabilidad } \\
\text { Trabajo en }\end{array}$ & $40 \%$ & $42 \%$ & $71 \%$ & $47 \%$ & $50 \%$ \\
$\begin{array}{c}\text { equipo } \\
\begin{array}{l}\text { Disposición al } \\
\text { aprendizaje }\end{array}\end{array}$ & $25 \%$ & $39 \%$ & $71 \%$ & $65 \%$ & $53 \%$ \\
$\begin{array}{c}\text { Orientación a los } \\
\text { resultados }\end{array}$ & $26 \%$ & $50 \%$ & $71 \%$ & $38 \%$ & $46 \%$ \\
$\begin{array}{l}\text { Comunicación } \\
\text { GLOBAL }\end{array}$ & $30 \%$ & $25 \%$ & $71 \%$ & $29 \%$ & $41 \%$ \\
\hline
\end{tabular}

Resultados en porcentajes de la evaluación $360^{\circ}$ al educando de Administrativo - Contable - 1er Periodo 2017 Fuente: Elaboración propia a partir de los datos proporcionados por el departamento de autoevaluación y calidad.

Tabla 2.

\begin{tabular}{lccccc}
\hline n=121 & $\begin{array}{c}\text { Evaluació } \\
\text { n docente }\end{array}$ & Autoevaluació & Heteroevaluació & Coevaluació & Promedi \\
$\mathbf{n}$ & $61 \%$ & $63 \%$ & $89 \%$ & $71 \%$ & $71 \%$ \\
\hline $\begin{array}{c}\text { Adaptabilidad } \\
\text { Trabajo en }\end{array}$ & $59 \%$ & $73 \%$ & $89 \%$ & $84 \%$ & $76 \%$ \\
$\begin{array}{c}\text { equipo } \\
\text { Disposición al }\end{array}$ & $71 \%$ & $65 \%$ & $89 \%$ & $62 \%$ & $72 \%$ \\
$\begin{array}{c}\text { aprendizaje } \\
\text { Orientación a los }\end{array}$ & $66 \%$ & $78 \%$ & $89 \%$ & $68 \%$ & $75 \%$ \\
$\begin{array}{l}\text { resultados } \\
\text { Comunicación }\end{array}$ & $78 \%$ & $73 \%$ & $89 \%$ & $79 \%$ & $80 \%$ \\
GLOBAL & & & & & $75 \%$ \\
\hline
\end{tabular}

Resultados en porcentajes de la evaluación $360^{\circ}$ al educando de Administrativo - Contable - 2do Periodo 2017

Fuente: Elaboración propia a partir de los datos proporcionados por el departamento de autoevaluación y calidad.

En los resultados obtenidos se puede evidenciar que el proceso conllevo un análisis de 4 aspectos fundamentales, los mismos que son parte de la evaluación $360^{\circ}$. Estas categorías son la evaluación docente que consiste en el resultado numérico que dispone cada docente sobre el desarrollo de las competencias de cada uno de los alumnos. La autoevaluación que consiste en la valoración propia que tiene cada miembro del educando con respecto a su propio rendimiento (en las tablas 1 y 2 se presenta un promedio general).

La heteroevaluacion es la evaluación del rendimiento enfocado en el desarrollo exclusivamente cognitivo, es decir al desarrollo que el alumno tiene con respecto a las asignaturas que se le proporcionan, por tal razón se coloca un promedio general de $71 \%$ en la 
tabla 1 y $89 \%$ en la tabla 2, como referencia al resultado global de los tres paralelos. Por último, tenemos la coevaluación que consiste en la evaluación de competencias que los alumnos hacen a sus pares, para esto se procede a una evaluación entre pares de compañeros de grupos con los que hayan trabajado en mínimo 3 ocasiones (Jiménez et al., 2010); para en el final tener un promedio global que se muestra como resultado y alcance del indicador sobre un $100 \%$.

En el comparativo de las tablas 1 y 2 ; existe un incremento del $30 \%$ en la tabla 2, es decir posterior a la implementación del proceso Coaching educativo. Este incremento general obedece al crecimiento de los factores obtenidos en el proceso de evaluación $360^{\circ}$; en la evaluación docente se presenta un incremento de $36 \%$; en la autoevaluación de 32\%; en la heteroevaluación de $18 \%$ y en la coevaluación de $32 \%$. Todo esto, en relación a los tres paralelos que componen la carrera.

\section{Discusión}

El análisis de artículos y libros sobre Coaching y sobre Coaching educativo brindan una gran cantidad de información relevante para los estudios de la gestión por competencias en el aula, lo que permite al proceso de enseñanza - aprendizaje una mejora continua en relación a la utilización de metodologías que benefician el desenvolvimiento académico. Además, es importante señalar que los procesos de Coaching no solo tienen un foco de atención en el educando; más bien, su desarrollo está orientado en mejorar y potencializar las competencias técnicas y conductuales de cualquier individuo, en cualquier ámbito; sea este empresarial, familiar, deportivo, etc.

La presente información hace referencia al análisis de la implementación de un proceso de Coaching educativo desde un análisis experimental, con el fin de determinar si este proceso puede influir en la gestión por competencias de un grupo de alumnos de determinada carrera e institución. Para esto, se tienen resultados positivos mediante una herramienta de análisis integral como lo es la evaluación $360^{\circ}$ que en este caso está enfocada netamente en medir el desarrollo del educando, mediante los elementos de evaluación docente, autoevaluación, heteroevaluación y coevaluación.

Siguiendo este orden de ideas, tenemos que también existe un alto nivel de desconocimiento sobre el Coaching en el área educativa, ya que en muchos de los casos solo se lo relaciona con el ámbito deportivo y/o empresarial (García-Naveira et al., 2017). Además, como se mencionó en anteriores párrafos, existen entidades públicas y privadas que realizan el proceso de certificación Coaching mediante instituciones y organismos evaluadores que permiten que determinada persona adquiera las competencias para poder ejercer la actividad de Coach, por ello es recomendable que la persona que decida ejercer esta actividad, en cualquier área, posea conocimientos sólidos y la práctica suficiente para la alianza estratégica y el logro de los objetivos del Coachee.

La utilización de los procesos de Coaching educativo en diferentes sectores, comunidades y países son evidentes; además es notable el desarrollo de las competencias del educando objeto de estudio, tal es el caso de un institución en Toluca - México; en ella se llevó a cabo un estudio cualitativo en estudiantes de formación superior de educación básica, este estudio tuvo como finalidad demostrar que la implementación del Coaching educativo, enfocado en el desarrollo de competencias del alumno, contribuye en mejorar el rendimiento académico. En este caso, el 
proceso se lo realizó como un modelo de acompañamiento más el proceso de implementación de preguntas poderosas.

El resultado del proceso; corroborado con una evaluación, la que conlleva la herramienta de medición TMMS-24 de inteligencia emocional, la prueba TMD que mide la orientación a objetivos y el test de Honey y Mumford que evalúa los estilos de aprendizaje; dio como resultados un impacto positivo sobre la implementación del Coaching, por lo que en la primera conclusión de aquel estudio se afirma que el Coaching educativo potencializa las competencias del educando, aumentando su capacidad de aprendizaje, su habilidad de resolver problemas y mejora su motivación (Arzate, 2013).

Un segundo caso es de origen europeo, específicamente en Girona-España, el proceso de Coaching se realizó a estudiantes de maestría en gestión de la calidad, a los cuales se les entregó la misión de elaborar un propio modelo de gestión, para lo cual ellos iban a tomar todas las directrices, los métodos de trabajo, los equipos, los roles y decisiones del caso; el cuerpo docente solo se limitaba a realizar procesos de acompañamiento y de Coaching en el que no intervenían para ordenar acciones; más bien sólo para escuchar activamente, realizar preguntas poderosas, modelos de causa/efecto que permitan descubrir y alcanzar soluciones al proyecto con total autonomía y autogestión del alumno. Los resultados de este proceso fueron muy efectivos ya que todos los alumnos de aquel proyecto pasaron el módulo y además mejoraron sus habilidades investigativas y de trabajo en equipo. Posterior a ello se presentó un aumento del $30 \%$ de empleabilidad en aquellos graduados (Piñeiro et al., 2013).

En sí, tomando en consideración nuestro estudio, el mismo se orienta a una evaluación de competencias que obedecen a una metodología de gestión de talento humano propuesto por Martha Alles (Alles, 2009), ya que los contenidos mencionados en aquella obra tienen relación a los utilizados en nuestro medio latinoamericano. Además, la elección de las competencias de análisis de la evaluación $360^{\circ}$ utilizada por la institución, está afinada a sus competencias organizacionales.

\section{Conclusiones}

Los elementos que dan comienzo a la terminología Coaching, son oriundos de Europa, desde un carruaje hasta un verbo de acción; sin embargo el desarrollo de la educación, la Psicología del aprendizaje y Pedagogía, han presentado bases científicas que se han adaptado al Coaching, ya que este es un proceso de formación, desarrollo, aprendizaje y sobre todo se enfoca en el desarrollo de competencias técnicas y conductuales de los sujetos.

Con respecto a la teoría, el Coaching se define como un proceso que desarrolla y potencializa las competencias de un sujeto por medio de una serie de determinados pasos, que van desde el análisis hasta la acción, por tal razón en esta investigación se corrobora que, en los casos de los alumnos trabajados en esta temática, si existe un incremento en sus competencias académicas que posteriormente se trasformarán en competencias laborales; por ende el Coaching se puede considerar una estrategia de efectividad en el desarrollo continuo de las competencias de los individuos (Devine et al., 2013). El objetivo del presente estudio oscila en identificar el desarrollo de competencias académicas a través de la implementación del Coaching educativo en los procesos de enseñanza aprendizaje del educando de nivel superior; por tanto, se puede afirmar que este proceso desempeña un papel formador y trasformador, orientado a potencializar 
las competencias y comportamientos del alumnado, siguiendo una serie de pasos establecidos y sincronizados de manera estratégica.

La utilización del método experimental tipo pre-experimental, es significativo y beneficioso por su claridad de abordaje mediante el análisis comparativo de un fenómeno en dos momentos de tiempo determinado. Por otra parte, es considerable mencionar que al momento no está disponible una herramienta específica y objetiva que mida el efecto del Coaching en determinada áreas, sin embargo la evidencia de los resultados se lo puede tener por medio de los desenlaces de otras herramientas que cumplen un alto nivel de confiabilidad y validez, tal es el caso de los estudios de clima laboral, clima educativo, evaluación de $360^{\circ}$ etc. Además, en el momento se mantienen en experimentación futuras herramientas para validar el impacto del Coaching en los ámbitos laborales, educativos y de otra índole.

La presente temática, además del Coaching educativo, hace referencia a la problemática de modelos pedagógicos cerrados y a la transición de paradigmas educativos versus las nuevas modalidades de procesos de enseñanza - aprendizaje (Jiménez, 2012), en el cual el proceso Coaching presenta herramientas internas y externas de mucha utilidad práctica para el logro de objetivos de alumnos y docentes (Sánchez-Teruel, 2013). Las limitaciones del estudio radican en que el caso permitió analizar el desenvolvimiento académico de la muestra mencionada con antelación desde una perspectiva general, sin embargo una de las modalidades más utilizadas de Coaching es la de Coaching individual, ya que esta permite un proceso más personalizado que permite un análisis más complementario. Dentro del estudio también se presentó algún grado de resistencia al cambio por parte de algunos miembros del educando, pero esta situación fue girando de forma favorable en torno al desarrollo del procedimiento.

En fin, los datos obtenidos en el presente documento obedecen a un análisis preexperimental y de recopilación de información de los últimos años, por tal razón es considerable profundizar la literatura y las investigaciones sobre la temática, en especial en nuestro medio; haciendo énfasis de los procesos de Coaching educativo en: la práctica docente, departamentos de bienestar estudiantil, e coordinaciones y directivas académicas (Rodríguez-Hidalgo et al., 2015); para así desarrollar de forma cuantitativa y cualitativa más estudios sobre la temática con el fin de analizar su relación con otras variables educativas.

\section{Bibliografía}

Alles, M. (2009). Diccionario de competencias. Buenos Aires: Ediciones Granica S.A.

Arzate, O. (2013). Coaching educativo: una propuesta metodológica para innovar en el aula. Ra Ximhai, 9(4), 177-185.

Bou-Pérez, J. (2007). Coaching para docentes. San Vicente - Alicante: Editorial Club Universitario.

Bravo, C. (2013). Pedagogía General (Vol. 1). Quito: Pontifica Universidad Católica del Ecuador.

Cubero, H., Visbal, E., \& Olivar, J. (2017). Propuesta para gestionar cambios de evaluación del desempeño. Caso: Universidad Simón Bolívar. Orbis. Revista Científica Ciencias Humanas, 13(38), 19-34.

Devine, M., Meyers, R., \& Houssemand, C. (2013). How can coaching make a positive impact within educational settings? Procedia, 93, $1382 \quad-\quad 1389$. doi:https://doi.org/10.1016/j.sbspro.2013.10.048 
García-Naveira, A. (2013). Aplicación profesional del coaching en el deporte: un estudio de caso único. Cuadernos de Psicología del Deporte, 13(2), 101-112.

García-Naveira, A., García-Mas, A., Ruiz-Barquín, R., \& Cantón, E. (2017). Programa de intervención basada en el coaching en jóvenes deportistas de alto rendimiento, y su relación con la percepción de bienestar y salud psicológica. Revista de Psicología del Deporte, 26(2), 37-44.

Giménez, J., Fleta, Y., \& Meya, A. (2016). Coaching nutricional para la pérdida de peso. Nutrición Hospitalaria, 33(1), 135-147.

Gómez, A. (2012). Fundamentos Pedagógicos de la educación (1a ed.). Sevilla: Universidad Pablo Olavide.

González, K., \& Esteban, C. (2013). Caracterización de modelos pedagógicos en formación elearning. Revista Virtual Universidad Católica del Norte (39), 4-16.

Gorrochotegui-Martell, A. (2011). Un modelo de "coaching" en directivos escolares. Educ.Educ., 14(2), 369-387.

Gorrochotegui-Martell, A., Vicente-Mendoza, I., \& Torres-Escobar, G. (2014). Evaluación de un proceso de coaching en directivos y su impacto en el clima escolar. Educación y Educadores, 17(1), 111-131.

Hernández Sampieri, R., Fernández, C., \& Baptista, M. (2014). Metodología de la Investigación (Vol. 6). México: McGraw-Hill.

Instituto Tecnológico Superior Vicente Rocafuerte. (1 de Agosto de 2017). http://itsvr.edu.ec/web1/mision-y-vision/. Obtenido de http://itsvr.edu.ec/web1/mision-yvision/

Isidoria, E., Miglioratia, M., Maulinia, C., \& Ramos-Echazarretaa, R. (2015). Educational Paradigms and Philosophy of Football Coaching: a Theoretical and Practical Perspective. Procedía, 197, 614-621. doi:https://doi.org/10.1016/j.sbspro.2015.07.203

Jiménez, R. (2012). Coaching en el desarrollo profesional docente. Fases formativas y procesos metodológicos de investigación. Revista Electrónica Interuniversitaria de Formación del Profesorado, 15(4), 101-112.

Jiménez, Y., González, M., \& Hernández, J. (2010). Modelo $360^{\circ}$ para la evaluación por competencias (enseñanza-aprendizaje). Innovación Educativa, 10(53), 43-53.

Lárez, J. (2008). El coaching educativo como estrategia para potenciar el éxito durante la etapa de desarrollo y culminación del trabajo especial de grado. Sapiens. Revista Universitaria de Investigación, 9(2), 219-234.

López Villafranca, P., \& Gómez de Travesedo, R. (2016). Coaching académico para adquirir competencias profesionales. Estudio de caso en la Universidad de Málaga. Opción, 32(10), 95-110.

Malagón, F. (2011). Coaching educativo y académico: un nuevo modo de enseñar y aprender. Educación y Futuro (24), 49-66.

Máñez, C., Navarro, B., \& Bou, J. (2008). Coaching para docentes. (pág. 105). México: CSI.F.

Músico, G. (2015). Coaching: conciliación de vida laboral-personal (WLB). Revista del Centro de Investigación Universidad La Salle, 11(44), 121-142.

Ortiz, A., Sánchez, J., \& Sánchez, I. (2015). Los modelos pedagógicos desde una dimensión psicológica-espiritual. Revista Científica General José María Córdova, 13(15), 183-194.

Ospina, Y. (2013). La pedagogía y su incidencia en la formación de sujetos. Hallazgos, 157-170. doi:http://dx.doi.org/10.15332/s1794-3841.2013.0020.10. 
Pimentel, L., \& Rodríguez, A.-M. (2016). El coaching educativo para mejorar la motivación de los docentes. EDUNOVATIC2016 Congreso Virtual Internacional de Educación, Innovación y TIC (pág. 10). Granada: Redine.

Piñeiro, M., Martínez, M., \& Guillén, E. (2013). Planificación y organización de la docencia enseñar y aprender mediante coaching educativo. Univest, 1-7.

Recalde, H., Cantero, W., \& Jara, J. (2013). Globalización de la educación superior en Paraguay. Revista Gestão Universitária na América Latina - GUAL, 6(4), 37-48.

Rodríguez-Hidalgo, A., Calmaestra, J., \& Maestre, M. (2015). Desarrollo de competencias en el practicum de maestros: ABP y Coaching Multidimensional. Profesorado. Revista de Currículum y Formación de Profesorado, 19(1), 414-434.

Rosa, G., Riberas, G., Navarro-Segura, L., \& Vilar, J. (2015). El Coaching como Herramienta de Trabajo de la Competencia Emocional en la Formación de Estudiantes de Educación Social y Trabajo Social de la Universidad Ramón Llull, España. Formación Universitaria, 8(5), 77-89. doi:http://dx.doi.org/10.4067/S0718-50062015000500009

Ruiz Fernández, M. I., Boada-Grau, J., Merino Tejedor, E., \& Ficapal-Cusí, P. (2014). Una experiencia de coaching en estudiantes universitarios. International Journal of Developmental and Educational Psychology, 4(1), 515-518.

Ruiz, E., Gago, M., García, C., \& López, S. (2012). Recursos humanos y responsabilidad social corporativa (1a ed.). Madrid: McGraw-Hill.

Sánchez, B., \& Boronat, J. (2014). Coaching educativo: modelo para el desarrollo de competencias intra e interpersonales. Educación XX1, 17(1), 221-242. doi:http://dx.doi.org/10.5944/educxx1.17.1.1072

Sánchez, J., Lorenzo, A., Jiménez, S., \& Lorenzo, J. (2017). El entrenador como mentor de jugadores en formación: un estudio de relaciones entrenador-deportista positivas. Revista de Psicología del Deporte, 26(1), 95-99.

Sánchez-Teruel, D. (2013). El coaching pedagógico dentro del sistema educativo: innovando procesos. Revista Intercontinental de Psicología y Educación, 15(2), 171-191.

Sandoval, C., \& López, O. (2017). Educación, psicología y coaching: un entramado positivo. Educatio Siglo XXI, 35(1), 145-164. doi:http://dx.doi.org/10.6018/j/286261

Sans, M. (2012). ¿Qué es el coaching? Sus Orígenes, definición, distintas metodologías y principios básicos de actuación de un coach. Revista de investigación 3Ciencias, 1-11.

Universidad de Castilla-La Mancha. (25 de abril de 2016). https://www.uclm.es/. Obtenido de https://www.uclm.es/home: https://www.youtube.com/watch?v=IqdTPsft0x0

Valer, L., \& Hernández, G. (2010). Corrientes pedagógicas contemporáneas. Lima: Editorial de la UNMSM.

Vásquez, A. (2012). Modelos pedagógicos: medios, no fines de la educación. Cuadernos de Lingüística Hispánica (19), 157-168.

Veliz, L., \& Paravic, T. (2012). Coaching educativo como estrategia para fortalecer el liderazgo en enfermería. Ciencia y Enfermería, 2(XVIII), 111-117. doi:http://dx.doi.org/10.4067/S0717-95532012000200012

Whitmore, J. (2003). Coaching: el método para mejorar el rendimiento de las personas. Barcelona: Paidós empresa. 